\title{
The half-life of ${ }^{212} \mathrm{Po}$
}

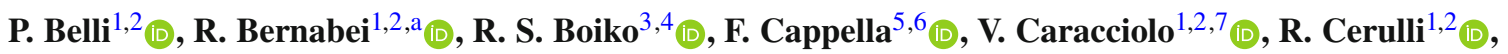

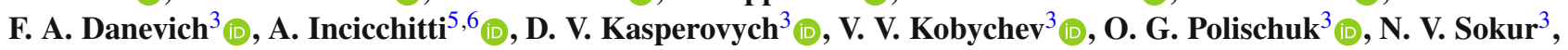 \\ V. I. Tretyak $^{3}$ (D) \\ ${ }^{1}$ INFN sezione Roma "Tor Vergata", 00133 Rome, Italy \\ 2 Dipartimento di Fisica, Università di Roma "Tor Vergata", 00133 Rome, Italy \\ ${ }^{3}$ Institute for Nuclear Research of NASU, Kyiv 03028, Ukraine \\ ${ }^{4}$ National University of Life and Environmental Sciences of Ukraine, Kyiv 03041, Ukraine \\ ${ }^{5}$ INFN sezione Roma, 00185 Rome, Italy \\ 6 Dipartimento di Fisica, Università di Roma "La Sapienza", 00185 Rome, Italy \\ ${ }^{7}$ Laboratori Nazionali del Gran Sasso, INFN, 67100 Assergi, AQ, Italy
}

Received: 10 February 2021 / Accepted: 30 May 2021 / Published online: 30 June 2021

(C) The Author(s) 2021

Communicated by Nicolas Alamanos

\begin{abstract}
The half-life of ${ }^{212}$ Po was measured with the highest up-to-date accuracy as $T_{1 / 2}=295.1$ (4) ns by using thorium-loaded liquid scintillator.
\end{abstract}

\section{Introduction}

The ${ }^{212}$ Po nuclide is the $\alpha$ active daughter of the ${ }^{212} \mathrm{Bi}$ from the ${ }^{232}$ Th decay chain with the shortest decay time among the naturally occurring radioactive nuclides. The decay scheme of the ${ }^{212} \mathrm{Bi} \rightarrow{ }^{212} \mathrm{Po} \rightarrow{ }^{208} \mathrm{~Pb}(\mathrm{BiPo})$ and ${ }^{212} \mathrm{Bi} \rightarrow{ }^{208} \mathrm{Tl}$ subchains is shown in Fig. 1. The current recommended value of the ${ }^{212}$ Po half-life is $T_{1 / 2}=294.3(8) \mathrm{ns}$ [1]. The history of the ${ }^{212}$ Po half-life measurements is presented in Table 1.

Gaseous counters have been used in the early experiments [3-8] to detect the $\beta$ particle emitted in the ${ }^{212} \mathrm{Bi}$ decay and the subsequent $\alpha$ particle of ${ }^{212}$ Po. Combinations of scintillation and semiconductor detectors were utilized in the experiments $[9,10,12,13]$, with $\beta$ and $\alpha$ particles from an external source (where the BiPo sequence of decays occurred) registered by the detectors.

A different approach has been exploited by using a liquid scintillator enriched with a source containing the BiPo chain [11]. A similar approach was exploited by the Borexino collaboration: quartz vials with thorium and ${ }^{220} \mathrm{Rn}$-loaded liquid scintillators were inserted into the Borexino Counting Test Facility (CTF) detector [14]. The half-life of ${ }^{212}$ Po was also measured in the experiment [15] with the help of a $\mathrm{BaF}_{2}$ crystal scintillator contaminated by radium. However, the accuracy of the experiment was limited by the comparatively

a e-mail: rita.bernabei@ roma2.infn.it (corresponding author) slow scintillation response of the $\mathrm{BaF}_{2}$ scintillator (effective scintillation decay time $\tau_{\text {eff }} \approx 0.6 \mu \mathrm{s}$ ) and a poor signal-tonoise ratio due to a rather modest light yield of the scintillator $\left(\simeq 10^{3}\right.$ photons/ $\left.\mathrm{MeV}_{\gamma}\right)$ [18]. In the XENON100 detector (a xenon liquid/gas time projection chamber) a ${ }^{220} \mathrm{Rn}$ source was used to calibrate it; using such calibration data, a further measurement of the ${ }^{212}$ Po half-life was obtained [16].

It should also be noted that the half-life of ${ }^{212}$ Po was measured in Refs. [19-25] as a by-product of the BiPo subchain analysis for different purposes (estimation of lowcounting detectors' internal contamination by thorium, calibration of detectors, study of low-lying states in $\alpha$-decaying nuclei). Typically the results are affected by large statistical uncertainties up to $17 \% \%^{1}$, and the systematic effects were not evaluated. Much larger statistics was gathered in recent measurement [26] where an external Th source was placed between plastic scintillators viewed by a photomultiplier (PMT) obtaining $T_{1 / 2}=294.09(7)$ ns. Unfortunately, systematic effects in the experiment were not estimated. In Ref. [27] a radon gaseous source was used to calibrate the xenon liquid/gas time-projection chamber of the PandaX-II experiment obtaining $T_{1 / 2}=297(6)$ ns with quite big uncertainty comparing with those in the last experiments in Table 1.

Considering the data in Table 1, the liquid scintillator loaded with Th radionuclides appears a very promising way to reduce the ${ }^{212}$ Po half-life uncertainty. However, although the measurements performed by the Borexino collaboration have profited of a fast scintillation signal, the measurement's

\footnotetext{
${ }^{1}$ It should be noted, however, a rather small uncertainty of the half-life value obtained in work [21]: $T_{1 / 2}=297(1) \mathrm{ns}$.
} 
Table 1 Historical view of the measured half-lives of ${ }^{212} \mathrm{Po}$

\begin{tabular}{|c|c|c|}
\hline Reference (year) & Experimental technique/compilation & Half-life (ns) \\
\hline [3] (1939) & Geiger-Müller counters, external source & $300(100)$ \\
\hline [4] (1943) & Geiger-Müller counters, external source & $260(40)$ \\
\hline [5] (1948) & Geiger-Müller counters, external source & $300(15)$ \\
\hline [6] (1949) & Geiger-Müller counters and proportional counters, external source & $304(4)$ \\
\hline [7] (1949) & Geiger-Müller counters, external source & $220(10)$ \\
\hline [8] (1953) & Geiger-Müller and proportional counters, external source & $290(10)$ \\
\hline [9] (1962) & Plastic scintillators, external source & $305(25)$ \\
\hline [10] (1963) & $\mathrm{CsI}(\mathrm{Tl})$ and plastic scintillators, external source & $305(5)$ \\
\hline [11] (1972) & Source in liquid scintillator & $302(6)^{1}$ \\
\hline [12] (1975) & Plastic scintillator, surface barrier $\mathrm{Au}-\mathrm{Si}$ detector, external source & $296(2)$ \\
\hline [13] (1981) & Surface barrier Au-Si and HP-planar Ge detectors, external source & $309(11)$ \\
\hline [14] (2013) & Source in liquid scintillator & $294.7(10)$ \\
\hline [15] (2014) & Source in $\mathrm{BaF}_{2}$ scintillator & $298.8(16)$ \\
\hline [16] (2017) & Source in liquid/gas Xe time projection chamber & $293.9(12)$ \\
\hline [17] (2018) & Source in liquid scintillator (1st stage of the current experiment) & 294.8(19) \\
\hline [1] (2020) & Nuclear Data Sheets compilation & 294.3(8) \\
\hline Present study & Source in liquid scintillator & 295.1(4) \\
\hline
\end{tabular}

${ }^{1}$ Average of the two values 300(8) ns and 304(8) ns reported in Ref. [11]

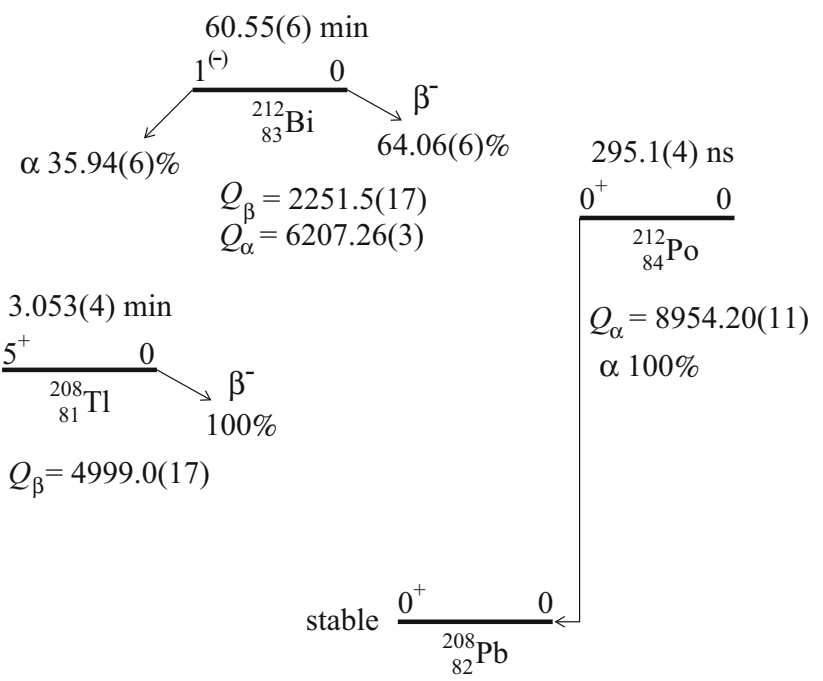

Fig. 1 The decay chain ${ }^{212} \mathrm{Bi} \rightarrow{ }^{212} \mathrm{Po} \rightarrow{ }^{208} \mathrm{~Pb}$ [1]. The $\alpha$ transition of ${ }^{212} \mathrm{Bi}$ to ${ }^{208} \mathrm{Tl}$ is shown too [2]. The half-life of ${ }^{212} \mathrm{Po}$ is from the present study. Energies of $\beta$ and $\alpha$ decays are given in $\mathrm{keV}$

precision was limited by the following aspects: the time jitter of the 100 photomultipliers array, scattering of the scintillation photons, their absorption and re-emission inside the CTF volume, and the readout electronics bandwidth. Moreover, a substantial difference $(\sim 40 \mathrm{~cm})$ in the photon paths to reach the PMTs has induced an additional time-spread distribution (average value of $2 \mathrm{~ns}$ ) due to the large volume of the CTF detector ( $4.8 \mathrm{~m}^{3}$ liquid scintillator).
According to all these observations, in order to improve the accuracy on the ${ }^{212}$ Po half-life measurements, one should minimize the liquid scintillator volume and use a fast response PMT/electronics. A thorium-loaded liquid scintillator (LS(Th)) was developed for the present study. Preliminary results of the measurements were published in [17]. Here we report the final result of the experiment with $\approx 64$ times larger statistics.

\section{Experiment}

\subsection{Thorium-loaded liquid scintillator}

\subsubsection{Production of thorium-loaded liquid scintillator}

Thorium nitrate pentahydrate $\mathrm{Th}\left(\mathrm{NO}_{3}\right)_{4} \cdot 5 \mathrm{H}_{2} \mathrm{O}$ was used as initial Th compound to prepare the $\mathrm{LS}(\mathrm{Th})$. A $20 \%$ solution of trioctylphosphine oxide (TOPO) in toluene was taken as complexing organophosphorous agent to bind thorium in organic phase. The mixture was stirred with thorium nitrate pentahydrate salt to obtain a solution containing $2 \mathrm{mg}$ of $\mathrm{Th}$ in $1 \mathrm{~mL}$ of TOPO solution:

$\mathrm{Th}^{4+}+4 \mathrm{NO}_{3}^{-}+3 \overline{\mathrm{TOPO}} \rightleftharpoons \overline{\mathrm{Th}\left(\mathrm{NO}_{3}\right)_{4}(\mathrm{TOPO})_{3}}$.

The obtained Th-containing organic solution was diluted 20 -fold with the liquid scintillator based on toluene with the addition of $0.1 \% 2.5$-diphenyl oxazole (PPO) and $0.01 \%$ 1,4-bis(5-phenyloxazol-2-yl) benzene (POPOP). Taking into 
account the preparation procedure, the liquid scintillator contains $\approx 0.1 \mathrm{wt} \%$ of thorium $\left({ }^{232} \mathrm{Th}\right.$ and ${ }^{228} \mathrm{Th}$ with daughters), while the activity of ${ }^{228} \mathrm{Ra}$ is expected to be rather low. Moreover, while ${ }^{228} \mathrm{Ra}$ has too small energy of $\beta$ decay $\left(Q_{\beta}=45.5(6) \mathrm{keV}\right.$ [28]) well below the hardware threshold (see Sect. 3.1), presence of some amount of ${ }^{228} \mathrm{Ac}$ ( $\beta$ active daughter of ${ }^{228} \mathrm{Ra}$ with the decay energy $Q_{\beta}=2123.8(26)$ $\mathrm{keV}$ [28]) leads to a mild contribution to the random-pairs background. Thus presence of some amount of ${ }^{228} \mathrm{Ra}$ in the scintillator almost does not affect the half-life of ${ }^{212}$ Po determination.

\subsubsection{Scintillation properties and activity of ${ }^{232}$ Th and its daughters in the $L S(T h)$}

A 7-mL sample of the LS(Th) was sealed inside a quartz vial with inner sizes $(\oslash 33 \times 14) \mathrm{mm}$ to test its scintillation properties and the activities of ${ }^{232} \mathrm{Th}$ and ${ }^{228} \mathrm{Th}$ with daughters. The vial was optically connected to a PMT Philips XP2412 and covered by $3 \mathrm{M}$ reflector foil to improve the scintillation-light collection. The signals from the PMT after a preamplification stage entered a shaping amplifier with $0.5 \mu$ s shaping time and then were processed by a peak sensitive analog-to-digital converter.

The relative light yield of the scintillator was estimated by using ${ }^{137} \mathrm{Cs}$ and ${ }^{207} \mathrm{Bi} \gamma$ sources to be $42(3) \%$ in comparison to a polystyrene based plastic scintillator $\oslash 30 \times 15 \mathrm{~mm}$ measured in the same conditions.

The energy spectrum measured with the LS(Th) is presented in Fig. 2. The energy scale of the detector was determined by analysis of the Compton electron spectra of the ${ }^{137} \mathrm{Cs}$ and ${ }^{207} \mathrm{Bi} \gamma$ sources as suggested in [29]. The peaks, in the (0.25-0.7) $\mathrm{MeV}$ energy range (see Fig. 2), can be attributed to $\alpha$ decays of ${ }^{232} \mathrm{Th}$ and ${ }^{228} \mathrm{Th}$ with daughters. The broad peak at $\sim 1.1 \mathrm{MeV}$ is due to the $\alpha$ decays of ${ }^{212}$ Po when the energy of the previously emitted $\beta$ particle is low. The overlap of $\beta$ events of ${ }^{212} \mathrm{Bi}$ and $\alpha$ events of ${ }^{212}$ Po produces the broad energy distribution up to $\sim 3.3$ $\mathrm{MeV}$ because the used acquisition did not separate these two events occurred in too short time in comparison to the amplifier shaping time.

To estimate the activity of ${ }^{232} \mathrm{Th}$ and ${ }^{228} \mathrm{Th}$ with daughters, the energy spectrum accumulated with the LS(Th) has been fitted by a model built from the $\alpha$ peaks of ${ }^{232} \mathrm{Th},{ }^{228} \mathrm{Th}$, ${ }^{224} \mathrm{Ra},{ }^{220} \mathrm{Rn},{ }^{216} \mathrm{Po}$ and ${ }^{212} \mathrm{Bi}$. The activities of the radionuclides, the energy resolution and the $\alpha / \gamma$ ratio $^{2}$ were free parameters of the fit. A linear function has been used to describe the distribution of $\beta$ particles and $\gamma$-ray quanta. Furthermore, taking into account the radon escape possibil-

\footnotetext{
${ }^{2}$ Defined as position of $\alpha$ peak in the energy scale measured with $\gamma$ quanta. Here we neglect the contribution of nuclear recoils because of a much higher quenching [30].
}

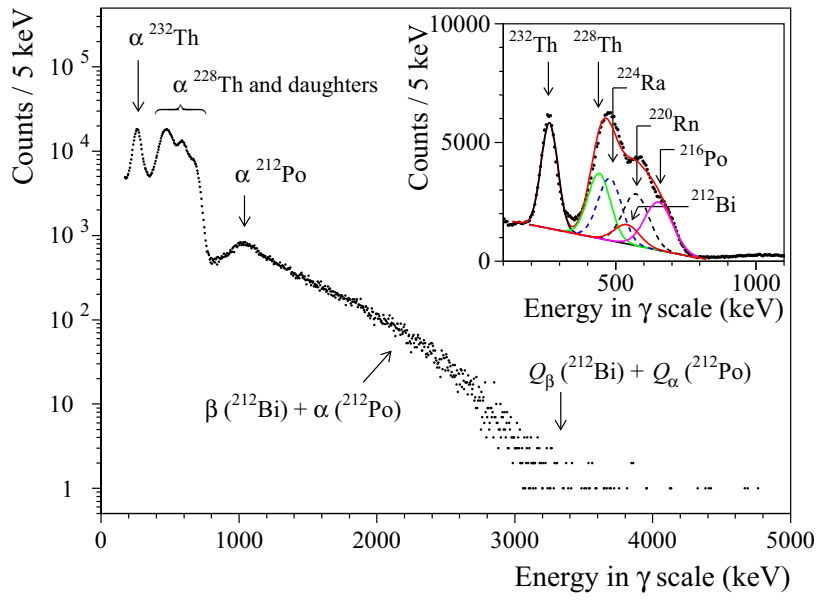

Fig. 2 Energy spectrum taken with $7 \mathrm{~mL} \mathrm{LS(Th)} \mathrm{scintillator} \mathrm{over}$ $5910 \mathrm{~s}$. The main features of the spectrum are shown. (Inset) Fit of the energy spectrum accumulated with the LS(Th) over $2050 \mathrm{~s}$ by a model which includes $\alpha$ peaks of ${ }^{232} \mathrm{Th}$ and ${ }^{228} \mathrm{Th}$ with daughters

ity from the LS(Th), we introduce a coefficient to consider a lower activity of ${ }^{220} \mathrm{Rn}$ and its daughters. The result of the fit in the energy interval $165-785 \mathrm{keV}\left(\chi^{2} /\right.$ n.d.f. $=1.45$, where n.d.f. is the number of degrees of freedom) is presented in the Inset of Fig. 2. The obtained behavior of the $\alpha / \gamma$ ratio in the energy range (4-9) $\mathrm{MeV}$ is described by the following formula: $\alpha / \gamma=0.02149(14)+0.01104(3) \times E_{\alpha}$ (where $E_{\alpha}$ is energy of the $\alpha$ particles in $\mathrm{MeV}$ ). The activities of ${ }^{232} \mathrm{Th}$ and ${ }^{228} \mathrm{Th}$ have been measured as 4.61(2) $\mathrm{Bq} / \mathrm{mL}$ and 3.82(7) $\mathrm{Bq} / \mathrm{mL}$, respectively. The lower activity of ${ }^{228} \mathrm{Th}$ is due to its decay after the thorium compound preparation in January 2016 (the measurements were performed on July 8th, 2016). The activity of the ${ }^{220} \mathrm{Rn}$ and its daughters is $92(2) \%$ of the ${ }^{228} \mathrm{Th}$ activity; this can be explained by radon escape from the $\mathrm{LS}(\mathrm{Th})$. The properties of the $\mathrm{LS}(\mathrm{Th})$ are summarized in Table 2.

\subsection{Recording of BiPo waveforms}

A sample of the LS(Th) in the same quartz vial was viewed by a fast-time-response PMT Hamamatsu R13089-100-11 with rise time $2.0 \mathrm{~ns}$, transit time $20 \mathrm{~ns}$ and transit time spread 170 ps (full width at half maximum, FWHM). The quartz vial was covered by polytetrafluoroethylene tape to improve the light collection. The signals waveforms were recorded by a LeCroy WavePro 735Zi-A oscilloscope with a sampling frequency $20 \mathrm{GSa} / \mathrm{s}$ and a $3.5 \mathrm{GHz}$ bandwidth.

The experiment was performed in two stages. In the first stage, carried out in June 2017, a $4.4 \mathrm{~g} \mathrm{(5} \mathrm{mL)} \mathrm{sample} \mathrm{of} \mathrm{the}$ liquid scintillator was utilized (the results of the first stage are published in [17]). A $10.6 \mathrm{~g}$ (12 mL) sample of the LS(Th) was used in the second stage of the experiment in AprilMay 2018. In total 785548 events were recorded in the first 
Table 2 Properties of the thorium-loaded liquid scintillator

\begin{tabular}{lll}
\hline Property & Value & Note \\
\hline Light yield & $42(3) \%$ & Relatively to polystyrene based plastic scintillator \\
Activity of ${ }^{232} \mathrm{Th}$ & $4.61(2) \mathrm{Bq} / \mathrm{mL}$ & \\
Activity of ${ }^{228} \mathrm{Th}$ & $3.82(7) \mathrm{Bq} / \mathrm{mL}$ & Reference date July 8th, 2016 \\
Total $\alpha$ activity & $20.7(10) \mathrm{Bq} / \mathrm{mL}$ & Reference date July 8th, 2016 \\
Concentration of thorium & $0.113(1) \mathrm{wt} \%$ & \\
\hline
\end{tabular}

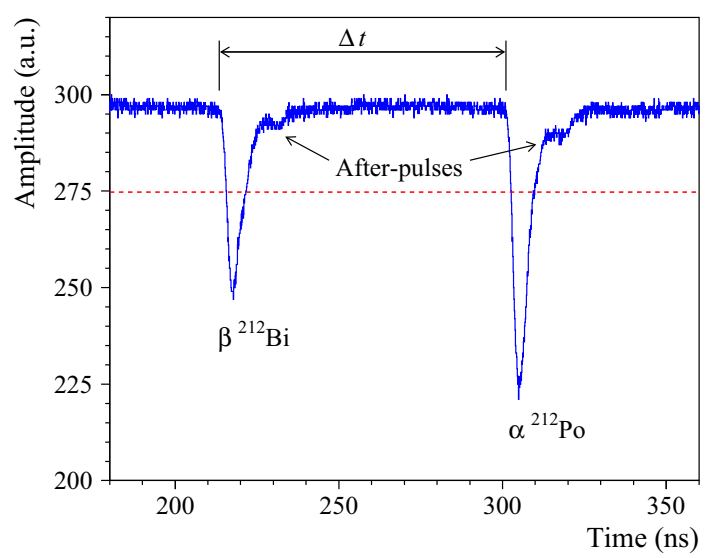

Fig. 3 Example of $\beta$ pulse of ${ }^{212} \mathrm{Bi}$ and subsequent $\alpha$ pulse of ${ }^{212} \mathrm{Po}$ in the liquid scintillator loaded by thorium. Dashed red line shows the software threshold used to identify pairs of events in the data. The time interval between the signals is denoted as $\Delta t$

stage, while 50340611 events (larger by a factor of $\approx 64$ ) were recorded in the second stage of the experiment during $216.67 \mathrm{~h}$ in order to improve the half-life value precision. The oscilloscope energy threshold in the second stage was set above the ${ }^{232} \mathrm{Th} \alpha$ peak in order to reduce the counting rate.

\section{Data analysis and results}

\subsection{Time intervals between $\beta$ and $\alpha$ pulses in BiPo events}

An example of a pair event in the LS(Th) classified as a BiPo event is shown in Fig. 3. After-pulses both for $\beta$ and $\alpha$ pulses of approximately 12-14 ns after the main pulses arise in the PMT because of the elastic scattering of the accelerated photoelectrons on the 1 st dynode. The scattered electrons return to the photocathode and then are multiplied again [31].

Amplitude spectra of the first and second events are shown in Fig. 4. The spectra were built by calculating the signals area, after baseline subtraction, in a time interval $11 \mathrm{~ns}$ (the time interval was chosen to avoid the effect of after-pulses). A constant was taken to describe the baseline of the first pulse, while a slow component of $\beta$ pulse (see Fig. 5 and discussion

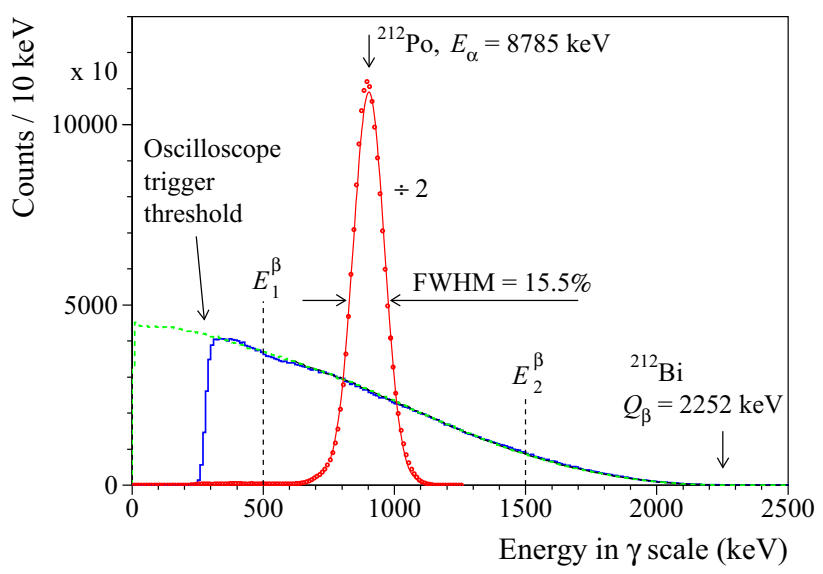

Fig. 4 Energy spectra of the 1 st events $\left(\beta\right.$ particles of ${ }^{212} \mathrm{Bi}$, solid histogram) and 2 nd events ( $\alpha$ particles of ${ }^{212}$ Po, dots). The Monte Carlo simulated response function of the detector to the $\beta$ decay of ${ }^{212} \mathrm{Bi}$ is shown by dashed histogram. The energy interval of $\beta$ events used in the analysis of the ${ }^{212}$ Po half-life is shown by vertical dashed lines labelled $E_{1}^{\beta}$ and $E_{2}^{\beta}$. The fit of the $\alpha$ peak by Gaussian function is shown by solid line. The $\alpha$ peak is divided by a factor of 2 to fit the figure

of the scintillation signals pulse shapes below) was added to the constant to reconstruct the second pulse shape. The detector energy scale was determined by comparison of the first events distribution with the Monte Carlo simulated $\beta$ spectrum of ${ }^{212} \mathrm{Bi}$. The response of the detector to the $\beta$ decay of ${ }^{212} \mathrm{Bi}$ was simulated using the GEANT4 package version 10.4.p02 (Shielding PEN physics list) [32-34] with initial kinematics given by the DECAY0 event generator $[35,36]$. The simulated-distribution shape reasonably agrees with the experimental data (see Fig. 4). The energy resolution of the $\mathrm{LS}(\mathrm{Th})$ detector was estimated for the $\alpha$ peak of ${ }^{212} \mathrm{Po}$ as $\mathrm{FWHM}=15.5 \%^{3}$. The obtained energy spectra confirm that the recognized pairs of events are mainly BiPo events.

In addition to the after-pulses observed 12-14 ns after the main pulses, a rather long sequence of pulses is visible in a sum of a big number of signals (see Fig. 5 where sums of

\footnotetext{
3 The difference in the ${ }^{212} \mathrm{Po} \alpha$-peak position in the spectra presented in Figs. 2 and 4 can be explained by different methods to build the spectra: a shaping amplifier and a peak sensitive analog-to-digital converter (the spectrum Fig. 2), and by using area of pulses for $<11 \mathrm{~ns}$ in the recorded waveforms (Fig. 4).
} 

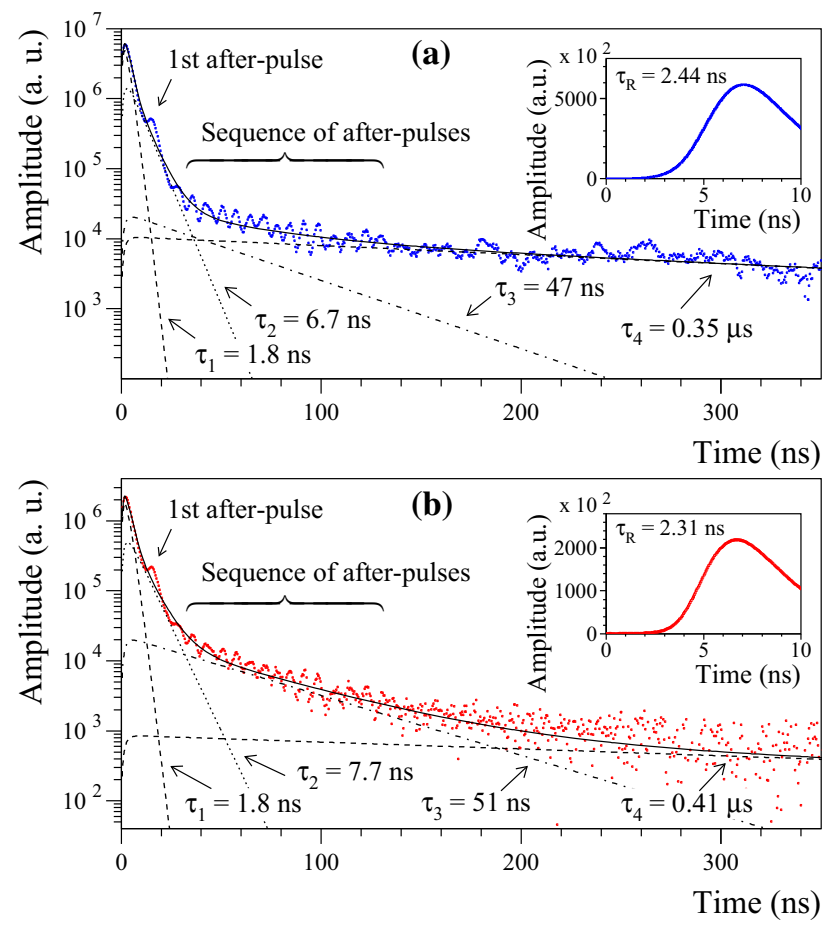

Fig. 5 Shape of scintillation pulses in the LS(Th) for $\beta$ particles of ${ }^{212} \mathrm{Bi}$ (a) and $\alpha$ particles of ${ }^{212} \mathrm{Po}$ (b). Fitting functions for $\beta$ and $\alpha$ pulses (solid lines), with four components of the scintillation signals (dashed, dotted and dash-dot lines) are shown. Zoomed $\beta$ and $\alpha$ pulses are shown in Insets. $\tau_{R}$ denotes the rise time of the pulses (see text for explanation)

about ten thousands $\beta$ pulses of ${ }^{212} \mathrm{Bi}$ and $\alpha$ pulses of ${ }^{212} \mathrm{Po}$ are presented). Explanation of the multiple after-pulses is problematic. However, we assume that this effect is somehow related to the operation of the photomultiplier. While the after-pulses have no impact on the determination of the 1st signal origin ( $\beta$-particle of ${ }^{212} \mathrm{Bi}$ ), they may affect the determination of the start time of the 2nd signal ( $\alpha$-particle of ${ }^{212} \mathrm{Po}$ ), and thus of the time interval between the two signals, especially for small $\Delta t<(30-60) \mathrm{ns}$.

The pulse shapes of $\beta$ and $\alpha$ scintillation signals are slightly different in the LS(Th). Fits of the pulses presented in Fig. 5 were done by the function:

$$
f(t)=\sum A_{i}\left(e^{-t / \tau_{i}}-e^{-t / \tau_{0}}\right) /\left(\tau_{i}-\tau_{0}\right), \quad t>0,
$$

where $A_{i}$ are the relative intensities, $\tau_{i}$ are the decay constants for different light-emission components, and $\tau_{0}$ is the time constant that describes the scintillation signal rise and integration of the photomultiplier and electronics $\left(\tau_{0} \approx 1.6(2)\right.$ ns both for $\beta$ and $\alpha$ pulses). Four decay components were observed with $\tau_{1} \approx 1.8 \mathrm{~ns}, \tau_{2} \approx 7 \mathrm{~ns}, \tau_{3} \approx 0.05 \mu \mathrm{s}$ and $\tau_{4} \approx 0.4 \mu$ s with different intensities for $\beta$ and $\alpha$ particles (see Table 3). It should be noted that the present investigation cannot be considered as analysis of the LS(Th) pulse-shape, in particular since the after-pulses were not excluded from the analysis. The estimations of the pulse-shape decay profile were performed to take into account the slow components of the $\beta$ pulses in the determination of the time intervals between $\beta$ and $\alpha$ pulses in BiPo events.

The following method was developed to find pairs of events in the data and determine the time intervals $\Delta t$ between the pulses:

(1) A simple low-level-discriminator algorithm with a high threshold (see Fig. 3) was applied to find pairs of events in the data.

(2) The recognized pairs of events were then analyzed by using the method of digital constant-fraction discrimination illustrated in Fig. 6. In the method the pulse-origin time (denoted in Fig. 6 as "Zero crossing time") was determined by analyzing the sum of two pulses produced from the recorded signal after the baseline subtraction: the first pulse was inverted and shifted in time by $11 \mathrm{~ns}$, and the second one integrated and multiplied by a factor 0.003 . The delay $11 \mathrm{~ns}$ was chosen to minimize effect of the undelayed pulse fluctuations on the timing (however, the delay was taken small enough to avoid possible afterpulses effect on the integrated pulse). The zero crossing time was found by fitting the sum pulse by exponential function around zero value. An example of the fit is shown in Fig. 6 too. The approach allows to eliminate an amplitude dependence of the pulse-time origin, which appears in the simple low-level-discriminator algorithm ${ }^{4}$.

The $\beta$ and $\alpha$ pulses have slightly different rise time ( $\tau_{R}$, defined as a time interval of the (10-90)\% rising edge): $\tau_{R}=2.44(16) \mathrm{ns}$ for $\beta$ particles and $\tau_{R}=2.31(14) \mathrm{ns}$ for $\alpha$ particles (see Insets in Fig. 5) ${ }^{5}$. The $\tau_{R}$ values were calculated for $\beta$ and $\alpha$ signals with amplitudes in the energy interval 700-1050 keV (in the energy scale of $\beta$ particles). This difference produces a systematic shift of $\Delta t$ that was estimated by using Monte Carlo simulations. The experimental data on the rise and trailing time distributions, and

\footnotetext{
${ }^{4}$ We have checked how the obtained half-life value (see below Sect. 3.2) depends on the constant-fraction discrimination method parameters. The data production was performed by using a constant-fraction discrimination method with the delay $1.8 \mathrm{~ns}$ and the multiplication factor 0.4 for the undelayed pulse (without integration). A fit of the obtained time distribution returns the half-life value $T_{1 / 2}=295.09(26) \mathrm{ns}$ in agreement with the value $T_{1 / 2}=295.10(26)$ ns obtained with the method described above.

${ }^{5}$ Rise time of scintillation detector depends on several factors: photodetector sensitivity and time properties, the readout electronics bandwidth, scintillation material, size and geometry of the scintillator and reflector, energy and ionization density of particle (see, e.g., [37-39]). The difference in the rise time for $\alpha$ and $\beta$ particles observed in the present study can be explained by their different energy distributions and ionization densities.
} 
Table 3 Decay time of the $\operatorname{LS}(\mathrm{Th})$ scintillator for $\beta$ and $\alpha$ particles. The decay constants and their relative intensities are denoted as $\tau_{i}$ and $\mathrm{A}_{i}$, respectively

\begin{tabular}{lllll}
\hline Type of events & \multicolumn{4}{l}{ Decay constants (ns) and relative intensities } \\
\cline { 2 - 5 } & $\tau_{1}, \mathrm{~A}_{1}$ & $\tau_{2}, \mathrm{~A}_{2}$ & $\tau_{3}, \mathrm{~A}_{3}$ & $\tau_{4}, \mathrm{~A}_{4}$ \\
\hline$\beta$ particles & $1.8(4)$, & $6.7(5)$, & $47(3)$, & $353(28)$, \\
& $56(10) \%$ & $32(4) \%$ & $2.7(2) \%$ & $9.3(4) \%$ \\
$\alpha$ particles & $1.8(4)$, & $7.7(6)$, & $51(3)$, & $409(134)$, \\
& $52(9) \%$ & $37(7) \%$ & $7.7(4) \%$ & $3.3(2) \%$ \\
\hline
\end{tabular}
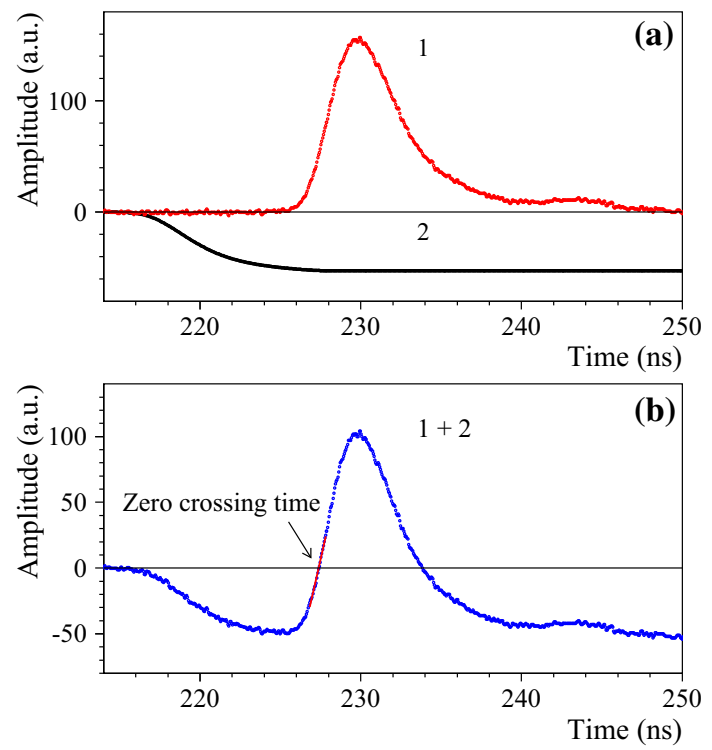

Fig. 6 Illustration of the digital constant-fraction discrimination method used to determine the signals starting time and to calculate $\Delta t$. a A scintillation pulse (after the baseline subtraction) inverted and shifted by $11 \mathrm{~ns}$ (1); the pulse integrated and multiplied by a factor 0.003 (2). b Sum of the pulses 1 and 2. The solid line shows the fit of the data by an exponential function. The arrow shows the zero crossing time accepted as the signal starting time

the energy spectra for $\beta$ and $\alpha$ events were taken as input parameters for the simulations. Noise was superimposed on the generated pulses. The noise was generated taking into account the baseline fluctuations of the recorded waveforms. In total $10^{4}$ double pulses were generated with $\Delta t=100$ ns. Analysis of the generated data by the constant-fractiondiscrimination algorithm returned $\Delta t=99.74 \mathrm{~ns}$. The difference $0.26 \mathrm{~ns}$ was added to the $\Delta t$ values for each BiPo event in the data production process.

Taking into account the difference of scintillation pulse shapes for $\beta$ and $\alpha$ particles, the mean-time method was applied to analyze the pulse profiles of the events. For each signal, the numerical characteristic of its shape (mean time, $\zeta$ ) was defined by using the following equation:

$\zeta=\sum f\left(t_{k}\right) \times t_{k} / \sum f\left(t_{k}\right)$
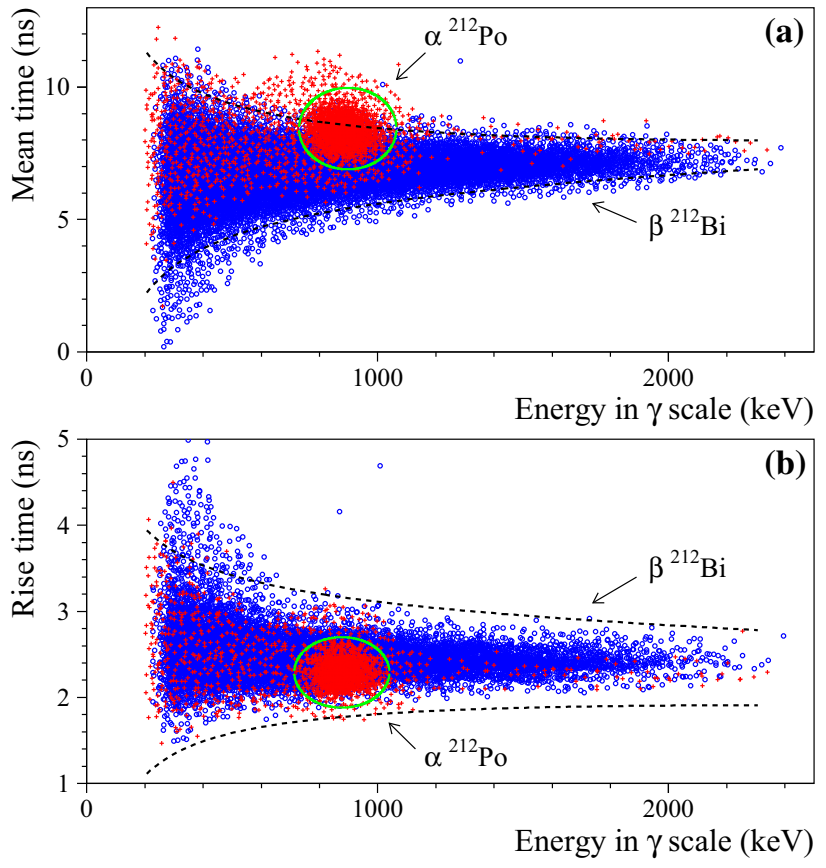

Fig. 7 Mean time (a) and rise time (b) vs energy distributions accumulated over $16.7 \mathrm{~h}$ with the $\mathrm{LS}(\mathrm{Th}$ ) (see text for explanation of the parameters). The $\pm 3 \sigma$ intervals for the mean-time and rise-time values for $\beta$ and $\alpha$ events selection are depicted by dashed lines (for $\beta$ events) and by solid curves in form of ellipses (for $\alpha$ particles)

where the sum is taken over the time channels $k$, starting from the origin of signal up to $60 \mathrm{~ns} ; f\left(t_{k}\right)$ is the digitized amplitude (at the time $t_{k}$ ) of a given signal.

The mean time vs energy distributions for the 1 st ( $\beta$ particles) and 2nd ( $\alpha$ particles) events selected from the data accumulated with the $\operatorname{LS}(\mathrm{Th})$ for $16.7 \mathrm{~h}$ are presented in Fig. 7a. We have used $\pm 3 \sigma$ regions both for $\beta$ particles of ${ }^{212} \mathrm{Bi}$ and for the $\alpha$ peak of ${ }^{212} \mathrm{Po}$ to select the BiPo events for the further analysis. The intervals to select $\beta$ and $\alpha$ events are shown in Fig. 7. Despite a rather poor particle discrimination ability, the filter reduces the contribution of random pairs of events and discards irregular-shape pulses of different origin.

The rise-time parameters for $\beta$ and $\alpha$ events were also analyzed (see Fig. 7b). All the pairs with $\beta$ or $\alpha$ pulses having the rise time outside the $\pm 3 \sigma$ regions were discarded from 

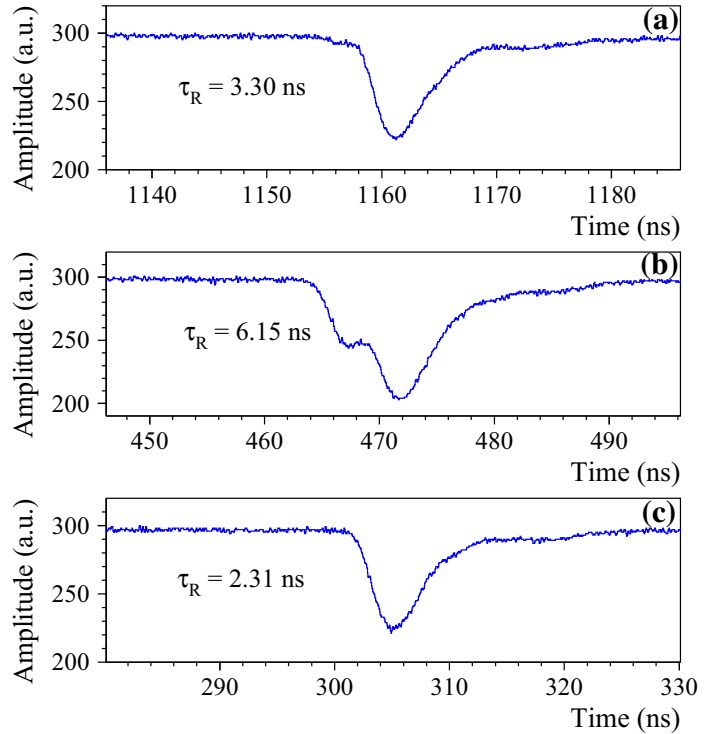

Fig. 8 Examples of $\alpha$ pulses with the rise-time values beyond the region of the accepted values for $\alpha$ particles (a and $\mathbf{b}$ ). A pulse with a normal rise time also shown c

the further analysis. Two examples of $\alpha$ pulses with abnormal rise-time values are shown in Fig. 8a, b together with a normal pulse (c). The pulses (a) and (b) with irregular shape can be explained by overlap of scintillation pulses or overlap of scintillation pulses with noise of different origin.

\subsection{Half-life of ${ }^{212} \mathrm{Po}$}

The distribution of the time intervals between the first and second signals in the recognized pairs of events is presented in Fig. 9. The pairs of events were selected with the first events amplitudes inside the energy interval $500-1500 \mathrm{keV}$ and with the mean-time and rise-time values inside the $\pm 3 \sigma$ bands shown in Fig. 7. The energy interval to select the first events ( $\beta$ particles of ${ }^{212} \mathrm{Bi}$ ) for the analysis is also shown in Fig. 4 (the choice of the energy interval for the 1st pulses selection will be explained below). The second events ( $\alpha$ particles of ${ }^{212} \mathrm{Po}$ ) were accepted inside the ellipses shown in Fig. 7.

The time distribution was fitted by a sum of two exponential functions: the first one describes the $\alpha$ decay of ${ }^{212} \mathrm{Po}$ and the second one is added to take into account random pairs of events [40]:

$N(t)=N_{1} e^{\left(-t \ln 2 / T_{1 / 2}\right)}+N_{2} e^{(-t b)}$,

where $N_{1}$ is proportional to the number of ${ }^{212} \mathrm{Po} \alpha$ decays selected, $T_{1 / 2}$ is the half-life of ${ }^{212} \mathrm{Po}, N_{2}$ is proportional to the number of random pairs of events, and $b$ is an average rate of random pairs. The random-pairs component (the parameters $N_{2}$ and $b$ ) was bounded in the energy intervals
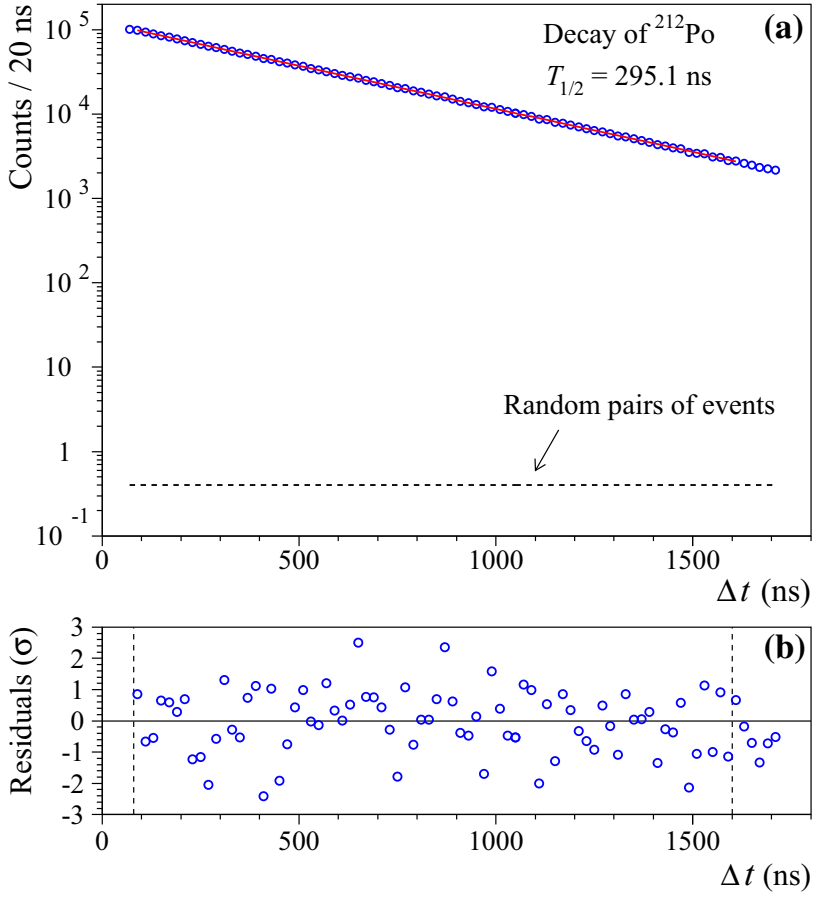

Fig. 9 a Distribution of the time intervals between the 1st and 2nd signals in the selected pairs of events $(\Delta t)$ and its fit by an exponential function with the half-life $T_{1 / 2}=295.1 \mathrm{~ns}$ plus background due to random pairs of events. The distribution of random pairs of events is shown too. $\mathbf{b}$ Residuals of the fit. The interval of fit is shown by vertical dashed lines

chosen for the analysis from the counting rate of the events taking into account the selection criteria for the mean-time and rise-time parameters. It should be stressed that the background due to the random pairs of events is very low in the present experiment: the parameters $N_{2}$ and $b$ were estimated to be $N_{2} \approx 3.3 \times 10^{-6} N_{1}$ and $b \approx 2.4 \mathrm{~s}^{-1}$. The distribution of random pairs of events is shown in Fig. 9a.

A fit of the $\Delta t$ distribution in the time interval 80-1600 ns by the maximum-likelihood method was performed with the help of the PAW [41] package that uses MINUIT software [42] for function minimization and uncertainty analysis. The fit returns the half-life of ${ }^{212}$ Po 295.10 (26) ns with $\chi^{2}=$ 77.4 for 73 degrees of freedom. The results of the fit are shown in Fig. 9. According to the recommendations [43], taking into account that the uncertainty of the half-life value was obtained by statistical methods, it is standard uncertainty obtained by type A evaluation method. Uncertainties due to possible systematic effects (Type B evaluation of standard uncertainty [43]) and a combined standard uncertainty of the result are reported in the next Section.

\subsection{Combined standard uncertainty}

The $T_{1 / 2}$ values obtained by the fit of the data with the bin widths from $0.05 \mathrm{~ns}$ (the oscilloscope time bin) to $100 \mathrm{~ns}$ lie 


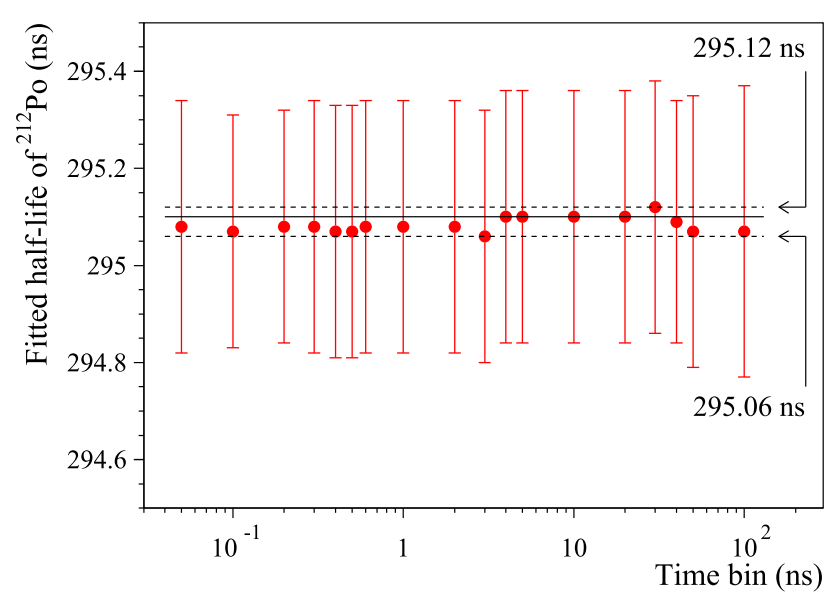

Fig. 10 The half-life of ${ }^{212} \mathrm{Po}$ as a function of the $\Delta t$ distribution timebin width. The value of the ${ }^{212}$ Po half-life $295.1 \mathrm{~ns}$ is shown by solid line, while the upper and lower values are shown by dashed lines

between $295.06 \mathrm{~ns}$ and $295.12 \mathrm{~ns}$ (see Fig. 10). Assuming a uniform distribution of possible $T_{1 / 2}$ values inside the interval, the standard deviation due to time-bin width, $0.02 \mathrm{~ns}$, was calculated as the upper value minus the lower value divided by the square root of 12 [43].

The result of fit depends on the low and high time bounds (see Fig. 11). The interval of fit (80-1600 ns) was chosen to minimize the combined uncertainty of the half-life value, taking into account that a bigger time interval is preferable to obtain smaller uncertainty due to statistical fluctuations. However, the point $60 \mathrm{~ns}$ was excluded from the analysis to reduce possible effect of after-pulses and slow components of the scintillation decay on the determination of the second pulse zero-crossing time ${ }^{6}$. Assuming a normal distribution of possible $T_{1 / 2}$ values, the standard deviations of the 15 half-life values in the time interval 80-360 ns were taken as uncertainties: $s_{1}={ }_{-0.22}^{+0.09} \mathrm{~ns}$ (the 7 points above the value $T_{1 / 2}=295.1 \mathrm{~ns}$ were taken to calculate the upper uncertainty, while the lower uncertainty was calculated by using the 7 points below the value). Similarly the standard deviations of the 15 fit results in the time interval 1400-1680 ns were taken as uncertainties due to the upper bound of the fit interval: $s_{2}={ }_{-0.06}^{+0.07} \mathrm{~ns}$. The intervals where the uncertainties were evaluated, as well as the lower and upper uncertainties, are shown in Fig. 11.

There is an indication of the half-life dependence on amplitude of the $\beta$ and $\alpha$ pulses. To estimate uncertainties due to the $\beta$-events amplitude, the lower energy threshold of $\beta$ events was varied within the energy interval 330-1500 $\mathrm{keV}$ with the steps from 170 to $500 \mathrm{keV}$ (see Fig. 12). The dependence of the half-life on the $\alpha$-signals amplitude was analyzed for the BiPo events with $\alpha$ events in the first and

\footnotetext{
${ }^{6}$ It should be noted that a much bigger effect of the fitted half-life increase at low time bounds was observed in the experiment [16].
}
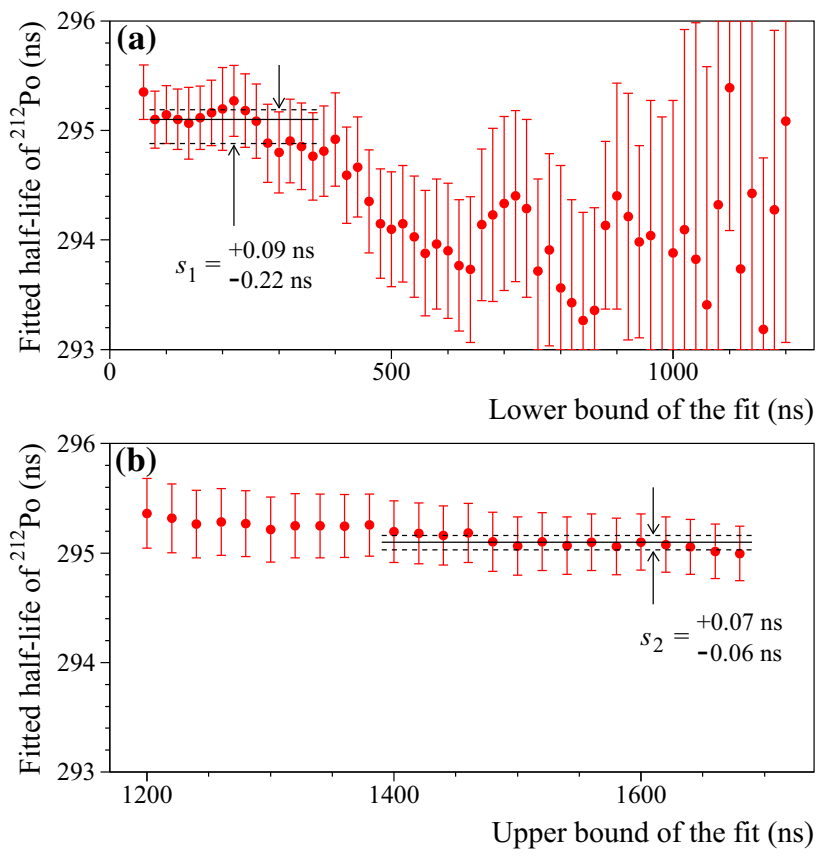

Fig. 11 The half-life of ${ }^{212}$ Po as a function of the lower (a) and higher bound (b) of the fit. $1600 \mathrm{~ns}$ was chosen as the end point for the fits presented in the panel (a), while the starting point was $80 \mathrm{~ns}$ for the fits shown in the panel (b). The standard deviations (shown by dashed lines) were calculated for 15 values in the time intervals $80-360 \mathrm{~ns}$ (denoted as $s_{1}$ ) and 1400-1680 ns $\left(s_{2}\right)$ to estimate the uncertainties of the lower and upper bounds of the fit. The value of the ${ }^{212}$ Po half-life $T_{1 / 2}=295.1 \mathrm{~ns}$ is shown by solid line

second halves of the $\alpha$ peak, while the $\beta$ events were in the energy interval $500-1500 \mathrm{keV}$. The effect could be explained by dependence of the PMT transit time on the pulse amplitude. The related uncertainties were estimated as standard deviations within the energy interval $500-1500 \mathrm{keV}$ for $\beta$ particles $\left(s_{\beta}={ }_{-0.16}^{+0.13} \mathrm{~ns}\right)$, and as the differences $\left(d_{\alpha}={ }_{-0.15}^{+0.16}\right.$ ns) between the half-life value $T_{1 / 2}=295.1 \mathrm{~ns}$ and the halflife values obtained for the two halves of the $\alpha$ peak. Eventually the energy interval $500-1500 \mathrm{keV}$ for the energy of $\beta$ particles was chosen for the analysis to reduce a possible effect of the half-life dependence on the pulses amplitude.

A possible effect of temperature variation during the data taking was checked by analyzing the two data sets gathered in the time intervals during the day with low (on the average $18^{\circ} \mathrm{C}$ for 3.9 hours around 4:42 AM) and high $\left(21^{\circ} \mathrm{C}\right.$ for 3.9 hours around 1:36 PM) temperatures in the room where the experimental set-up was installed. The analysis of the data sets returned the half-life values $T_{1 / 2}=295.66(64) \mathrm{ns}$ and $T_{1 / 2}=295.43(62)$ ns for the "low" and "high" temperature periods, respectively, that is no evidence for the effect. Nevertheless, a systematic uncertainty due to a possible temperature effects was estimated as the half of the difference between the two values: $\pm 0.12 \mathrm{~ns}$. 


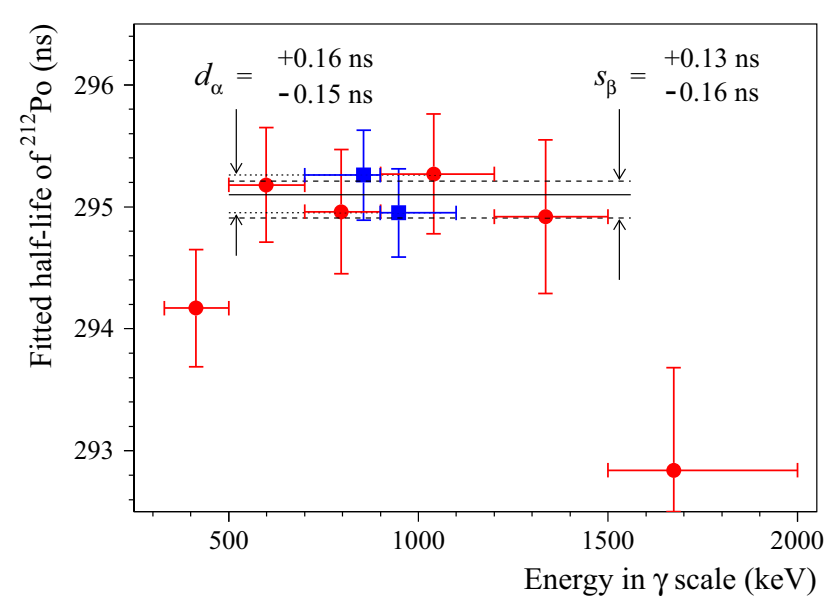

Fig. 12 The half-life of ${ }^{212} \mathrm{Po}$ as a function of the amplitude of the 1 st ( $\beta$ particles of ${ }^{212} \mathrm{Bi}$, dots) and 2 nd events ( $\alpha$ particles of ${ }^{212} \mathrm{Po}$, squares). Dashed lines show the uncertainties due to the $\beta$ pulses amplitude (denoted $s_{\beta}$ ), dotted lines show the uncertainties due to the $\alpha$ pulses amplitude $\left(d_{\alpha}\right)$

Table 4 Uncertainty evaluation of the half-life of ${ }^{212} \mathrm{Po}(\mathrm{ns})$

\begin{tabular}{ll}
\hline Standard deviation by statistical methods & \pm 0.26 \\
Lower bound of the fit & +0.09 \\
& -0.22 \\
Upper bound of the fit & +0.07 \\
& -0.06 \\
Amplitude of $\beta$ events & +0.13 \\
& -0.16 \\
Amplitude of $\alpha$ events & +0.16 \\
& -0.15 \\
Time bin & \pm 0.02 \\
Variations of temperature & \pm 0.12 \\
Uncertainty of the oscilloscope & \pm 0.0017 \\
Combined standard uncertainty & +0.37 \\
& -0.43 \\
\hline
\end{tabular}

Finally, uncertainty of the oscilloscope to measure time interval between two pulses was estimated as $\sqrt{2} \times S C J+$ $C A R$ [44], where $S C J$ is Sample Clock Jitter $(S C J=$ $0.0001 \mathrm{~ns}$ ) and $C A R$ is the product of the Clock Accuracy $(1 \mathrm{ppm})$ and the time stamp of the second pulse (conservatively we took the maximal time interval $1600 \mathrm{~ns}$ and got the value $C A R=0.0016$ ). Thus, we accept $0.0017 \mathrm{~ns}$ as an uncertainty due to a possible oscilloscope inaccuracy.

No other sources of uncertainty were observed. A summary of the uncertainties is given in Table 4 .

Treating all the listed in Table 4 uncertainties as independent and adding them in quadrature, we obtain the following half-life of ${ }^{212} \mathrm{Po}$ :

$T_{1 / 2}=295.1(4) \mathrm{ns}$.

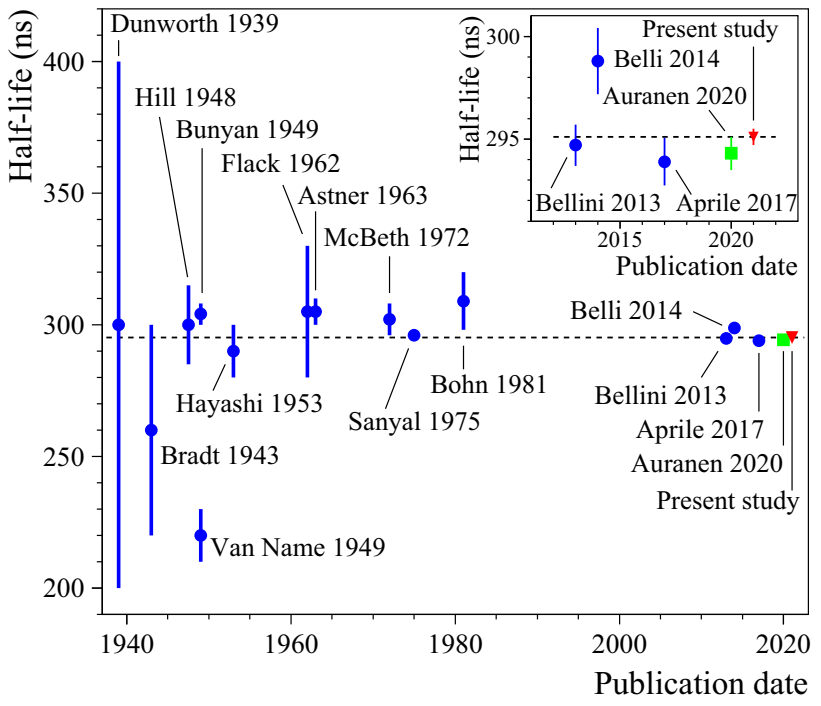

Fig. 13 A historical perspective of the half-life of ${ }^{212} \mathrm{Po}$ as a function of the publication date. References to the publications are as follows: Dunworth (1939): [3], Bradt (1943): [4], Hill (1948): [5], Bunyan (1949): [6], Van Name (1949): [7], Hayashi (1953): [8], Flack (1962): [9], Astner (1963): [10], McBeth (1972): [11], Sanyal (1975): [12], Bohn (1981): [13], Bellini (2013): [14], Belli (2014): [15], Aprile (2017): [16]. The recommended value Auranen (2020) [1] is shown by square; the result of the present study is shown by triangle. In the Inset the most recent measurements are reported

The present measurement is the most accurate determination of the ${ }^{212}$ Po half-life. The value is in agreement with the preliminary result of the previous stage of the present experiment $T_{1 / 2}=294.8(19)$ ns [17] and with the recommended value $T_{1 / 2}=294.3(8) \mathrm{ns}$ [1]. The result agrees with the recent experiments: $T_{1 / 2}=(294.7 \pm 0.6$ (stat.) \pm 0.8 (syst. $\left.)\right)$ ns [14] and $T_{1 / 2}=(293.9 \pm 1.0$ (stat.) \pm 0.6 (syst.) $)$ ns [16], but it is smaller than the one obtained in the measurement with $\mathrm{BaF}_{2}$ scintillation detector: $T_{1 / 2}=(298.8 \pm 0.8$ (stat. $) \pm$ 1.4(syst.)) ns [15]. A historical perspective of the half-life of ${ }^{212} \mathrm{Po}$ as a function of the publication date is presented in Fig. 13.

One can calculate a weighted average of the last half-life values [14-16], and of the present study as 295.1(4) ns, with the uncertainties combined in quadrature. If the value [15] is excluded, the weighted average becomes 294.9(4) ns.

\section{Conclusions}

The half-life of ${ }^{212}$ Po relative to $\alpha$ decay to the ground state of ${ }^{208} \mathrm{~Pb}$ (the only known channel of ${ }^{212}$ Po decay) was measured with thorium-loaded liquid scintillator as $T_{1 / 2}=295.1$ (4) ns. This result is the most accurate up-to-date value (relative uncertainty: $0.14 \%$ ). It has been achieved thanks to the utilization of the fast liquid scintillator (rise time does not exceed $\sim 1 \mathrm{~ns})$, its small volume $(12 \mathrm{~mL})$, the use of the 
fast photomultiplier with $\sim 2$ ns rise time and of the fast oscilloscope with a sampling frequency of $20 \mathrm{GSa} / \mathrm{s}$ and a $3.5 \mathrm{GHz}$ bandwidth, the high statistics of the acquired data, the developed algorithm to determine with a high accuracy the time intervals between the $\beta$ events of ${ }^{212} \mathrm{Bi}$ and the $\alpha$ events of ${ }^{212} \mathrm{Po}$.

Acknowledgements The group from the Institute for Nuclear Research of NASU (Kyiv, Ukraine) was supported in part by the National Research Foundation of Ukraine Grant No. 2020.02/0011. D.V. Kasperovych, O.G. Polischuk and N.V. Sokur were supported in part by the project "Investigations of rare nuclear processes" of the program of the National Academy of Sciences of Ukraine "Laboratory of young scientists".

Funding Open access funding provided by Università degli Studi di Roma Tor Vergata within the CRUI-CARE Agreement.

Data Availability Statement This manuscript has no associated data or the data will not be deposited. [Authors' comment: All data generated during this study are contained in this published article.]

Open Access This article is licensed under a Creative Commons Attribution 4.0 International License, which permits use, sharing, adaptation, distribution and reproduction in any medium or format, as long as you give appropriate credit to the original author(s) and the source, provide a link to the Creative Commons licence, and indicate if changes were made. The images or other third party material in this article are included in the article's Creative Commons licence, unless indicated otherwise in a credit line to the material. If material is not included in the article's Creative Commons licence and your intended use is not permitted by statutory regulation or exceeds the permitted use, you will need to obtain permission directly from the copyright holder. To view a copy of this licence, visit http://creativecomm ons.org/licenses/by/4.0/.

\section{References}

1. K. Auranen, E.A. McCutchan, Nuclear data sheets for $A=212$. Nucl. Data Sheets 168, 117 (2020)

2. M.J. Martin, Nuclear data sheets for $A=208$. Nucl. Data Sheets 108, 1583 (2007)

3. J.V. Dunworth, A determination of the half-value periods of Radium C' and Thorium C', with a note on time lags in a Geiger counter. Nature 144, 152 (1939)

4. H. Bradt, P. Scherrer, Die Halbwertszeit des ThC'. Helv. Phys. Acta 16, 259 (1943)

5. J.M. Hill, The half-Life of thorium C'. Proc. Camb. Phil. Soc. 44 440 (1948)

6. D.E. Bunyan, A. Lundby, D. Walker, Experiments with the delayed coincidence method, including a search for short-lived nuclear isomers. Proc. Phys. Soc. (Lond.) A 62, 253 (1949)

7. F.W. Van Name Jr., Measurement of the radioactive decay constant of ThC' using a coincidence method. Phys. Rev. 75, 100 (1949)

8. T. Hayashi, Y. Ishizaki, I. Kumabe, The half-life of ThC'. J. Phys. Soc. Jpn. 8, 110 (1953)

9. F.C. Flack, J.E. Jonson, The gamma radiation from ${ }^{212} \mathrm{Po}(\mathrm{ThC}$ '). Proc. Phys. Soc. 79, 10 (1962)

10. G. Astner et al., A hindered E2 ground state transition in ${ }^{207} \mathrm{Po}$. Nucl. Phys. 45, 49 (1963)

11. G.W. McBeth, R.A. Winyard, Isotope identification and radioassay by time interval analysis. Int. J. Appl. Rad. Isot. 23, 527 (1972)
12. S. Sanyal et al., Half-life measurement of the ${ }^{212}$ Po ground state. Phys. Rev. C 12, 318 (1975)

13. H. Bohn, E. Endres, T. Faestermann, P. Kienle, Spectroscopy of excited states in ${ }^{212} \mathrm{Po},{ }^{210} \mathrm{~Pb}$, and ${ }^{213}$ At employing ${ }^{18} \mathrm{O}$ induced few-nucleon transfer reactions. Z. Phys. A 302, 51 (1981)

14. G. Bellini et al., Lifetime measurements of ${ }^{214} \mathrm{Po}$ and ${ }^{212} \mathrm{Po}$ with the CTF liquid scintillator detector at LNGS. Eur. Phys. J. A 49, $92(2013)$

15. P. Belli et al., Investigation of rare nuclear decays with $\mathrm{BaF}_{2}$ crystal scintillator contaminated by radium. Eur. Phys. J. A 50, 134 (2014)

16. E. Aprile et al., Results from a calibration of XENON100 using a source of dissolved radon-220. Phys. Rev. D 95, 072008 (2017)

17. P. Belli et al., Half-life measurements of ${ }^{212} \mathrm{Po}$ with thorium loaded liquid scintillator. Nucl. Phys. At. Energy 19, 307 (2018). ((in Ukrainian))

18. Saint-Gobain Crystals, http://www.crystals.saint-gobain.com/. Accessed 22 May 2021

19. P. Belli et al., Performances of a $\mathrm{CeF}_{3}$ crystal scintillator and its application to the search for rare processes. Nucl. Instrum. Meth. A 498, 352 (2003)

20. F.A. Danevich et al., Search for $2 \beta$ decay of cadmium and tungsten isotopes: Final results of the Solotvina experiment. Phys. Rev. C 68, 035501 (2003)

21. R. Cerulli et al., Performances of $\mathrm{a} \mathrm{BaF}_{2}$ detector and its application to the search for $\beta \beta$ decay modes in ${ }^{130} \mathrm{Ba}$. Nucl. Instrum. Meth. A 525, 535 (2004)

22. P. Belli et al., Search for $\alpha$ decay of natural Europium. Nucl. Phys. A 789, 15 (2007)

23. P. Belli et al., Search for double- $\beta$ decay processes in ${ }^{106} \mathrm{Cd}$ with the help of a ${ }^{106} \mathrm{CdWO}_{4}$ crystal scintillator. Phys. Rev. C 85, 044610 (2012)

24. A. Samark-Roth et al., Low-lying states in ${ }^{219} \mathrm{Ra}$ and ${ }^{215} \mathrm{Rn}$ : Sampling microsecond $\alpha$-decaying nuclei. Phys. Rev. C 98, 044307 (2018)

25. A. Sonay et al., Neutron background measurements with a hybrid neutron detector at the Kuo-Sheng Reactor Neutrino Laboratory. Phys. Rev. C 98, 024602 (2018)

26. E.N. Alexeev et al., TAU-4 installation intended for long-term monitoring of a half-life value of the ${ }^{212}$ Po. arXiv:1812.04849 [physics.ins-det]

27. W. Ma et al., Internal calibration of the PandaX-II detector with radon gaseous sources. JINST 15, P12038 (2020)

28. M. Wang et al., The Ame 2020 atomic mass evaluation. Chin. Phys. C 45, 030003 (2020)

29. G. Dietze, H. Klein, Gamma-calibration of NE 213 scintillation counters. Nucl. Instrum. Meth. 193, 549 (1982)

30. V.I. Tretyak, Semi-empirical calculation of quenching factors for ions in scintillators. Astropart. Phys. 33, 40 (2010)

31. Communication from Hamamatsu Photonics K.K. https://www. hamamatsu.com/jp/en/3001.html. Accessed 22 May 2021

32. S. Agostinelli et al., GEANT4-a simulation toolkit. Nucl. Instrum. Meth. A 506, 250 (2003)

33. J. Allison et al., Geant 4 developments and applications. IEEE Trans. Nucl. Sci. 53, 270 (2006)

34. J. Allison et al., Recent developments in GEANT4. Nucl. Instrum. Meth. A 835, 186 (2016)

35. O.A. Ponkratenko et al., Event generator DECAY4 for simulating double-beta processes and decays of radioactive nuclei. Phys. At. Nucl. 63, 1282 (2000)

36. V.I. Tretyak (Institute for Nuclear Research of NASU, Kyiv, Ukraine). Private communication (2007)

37. L. Papadopoulos, Rise time of scintillation emission in scintillators. Nucl. Instrum. Meth. A 401, 312 (1997)

38. S.E. Derenzo et al., Measurements of the intrinsic rise times of common inorganic scintillators. IEEE Trans. Nucl. Sci. 47, 860 (2000) 
39. S.E. Derenzo et al., Fundamental limits of scintillation detector timing precision. Phys. Med. Biol. 59, 3261 (2014)

40. J. Radeloff, N. Buttler, W. Kesternich, E. Bodenstedt, Measurement of lifetimes up to one second of isomeric nuclear states populated in the decay of radioactive sources. Nucl. Instrum. Meth. 47, 109 (1967)

41. O. Couet et al., PAW - Physics Analysis Workstation, CERN Program Library Long Writeup Q12. Available at https://cdsweb.cern. ch/record/2296392/files/paw.pdf. Accessed 22 May 2021

42. F. James et al., MINUIT - Function Minimization and Error Analysis, CERN Program Library Long Writeup D506. Available at http://cdssls.cern.ch/record/2296388/files/minuit.pdf. Accessed 22 May 2021
43. Joint Committee for Guides in Metrology (JCGM) 100:2008, Evaluation of measurement data-Guide to the expression of uncertainty in measurement, https://www.bipm.org/utils/common/documents/ jcgm/JCGM_100_2008_E.pdf. Accessed 22 May 2021

44. Oscilloscope WavePro $7 \mathrm{Zi}$-A Series, Teledyne LeCroy, Inc (2017). Available at http://cdn.teledynelecroy.com/files/pdf/wavepro_7_ zi-a_datasheet.pdf. Accessed 22 May 2021 